\title{
CD1d- and PJA2-related immune microenvironment differs between invasive breast carcinomas with and without a micropapillary feature
}

\author{
Naoki Kanomata ${ }^{1 *}$ (D, Junichi Kurebayashi ${ }^{2}$, Yoshikazu Koike ${ }^{2}$, Rin Yamaguchi ${ }^{3,4}$ and Takuya Moriya ${ }^{1}$
}

\begin{abstract}
Background: Invasive micropapillary carcinoma (IMPC) of the breast is characterized by its unique morphology and frequent nodal metastasis. However, the mechanism for development of this unique subtype has not been clearly elucidated. The aim of this study was to obtain a better understanding of IMPC.

Methods: Using representative cases of mixed IMPC, mRNA expression in the micropapillary area and usual invasive area was compared. Then, immunohistochemical analyses for 294 cases (76 invasive carcinomas with a micropapillary feature [ICMF] and 218 invasive carcinomas without a micropapillary feature [ICNMF]) were conducted. Clinicopathological analyses were also studied.

Results: DNA microarray analyses for mixed IMPC showed that BC-1514 (C210rf118) was commonly upregulated in the micropapillary area. CAMK2N1, CD1d, PJA2, RPL5, SAMD13, TCF4, and TXNIP were commonly downregulated in the micropapillary area. Immunohistochemically, we confirmed that BC-1514 was more upregulated in ICMF than in ICNMF. CD1d and PJA2 were more downregulated in ICMF than ICNMF. All patients with cases of PJA2 overexpression survived without cancer recurrence during the follow-up period, although the differences for disease-free $(p=0.153)$ or overall survival $(p=0.272)$ were not significant.
\end{abstract}

Conclusions: The CD1d- and PJA2-related tumour microenvironment might be crucial for IMPC. Further study of the immune microenvironment and micropapillary features is warranted.

Keywords: Invasive micropapillary carcinoma, DNA microarray, CD1D, PJA2, Immunohistochemistry, Immune microenvironment

\section{Background}

Invasive micropapillary carcinoma (IMPC) of the breast is characterized by its unique morphology; formation of micropapillae within clear spaces separated by fibrous stroma, and reverse polarity [1]. A reverse polarity is shown by immunohistochemistry of EMA [2], MUC1 [3], sialyl Lewis X [4], and p120 catenin [5]. IMPC is known to have higher lymph vessel tumour embolus and nodal metastasis than invasive carcinoma of no special

* Correspondence: kanomata_7@med.kawasaki-m.ac.jp

${ }^{1}$ Department of Pathology, Kawasaki Medical School, Matsushima 577,

Kurashiki, Okayama 701-0192, Japan

Full list of author information is available at the end of the article type (ICNST) [2]. Tumour-infiltrating lymphocytes [6], p63 [7], involucre [7], 34ßE12 [7], stromal cell-derived factor-1 [8], CXCR4 [8], caveolin-1 [9], CD44 [10], prostate stem cell antigen [11], and LZTS1 [12] have been reported for IMPC. Marchiò et al. studied the comparative genomic hybridization analysis of pure IMPC [13] and concluded that high-level gain/amplification of 8p12-p11, 8q12, 8q13, 8q21, 8q23, 8q24, 17q21, 17q23, and $20 \mathrm{q} 13$ were significantly associated with IMPCs. However, to our knowledge, DNA microarray analysis of IMPC has not yet been reported. The aim of this study was to obtain better understanding of IMPC using DNA microarray analysis followed by immunohistochemistry.

(c) The Author(s). 2019 Open Access This article is distributed under the terms of the Creative Commons Attribution 4.0 International License (http://creativecommons.org/licenses/by/4.0/), which permits unrestricted use, distribution, and reproduction in any medium, provided you give appropriate credit to the original author(s) and the source, provide a link to the Creative Commons license, and indicate if changes were made. The Creative Commons Public Domain Dedication waiver (http://creativecommons.org/publicdomain/zero/1.0/) applies to the data made available in this article, unless otherwise stated. 


\section{Methods}

\section{DNA microarray}

After approval by the Institutional Review Board of Kawasaki Medical School ethics committee (approval number 909 and 2136), two representative cases of mixed IMPC were extracted from the database of the Department of Pathology, Kawasaki Medical School. Case 1 was ER-, PgR-, and HER2 3+, with histologic grade 2 and pT2N3aM0 (Fig. 1a and c). Case 2 was ER+, PgR-, and HER2-, with histologic grade 2 and pT1cN0M0 (Fig. 1b and $\mathrm{d}$ ). The micropapillary structure was confirmed with EMA and MUC1. Paraffin sections were cut from the blocks and deparaffinized. They were stained with toluidine blue. Under microscopy, the tissue was separated to the IMPC area and the ICNST area of each case by scalpal blade. RNA was extracted and hybridization on a 3D-Gene Human Oligochip (Toray, Tokyo, Japan). The microarray data from the IMPC and ICNST areas were compared.

\section{Antibodies}

Polyclonal antibodies were generated for SAMD13, TCF4 and TXNIP using synthetic peptides of their specific amino acid structures: positions 31-46 for SAMD13; positions 756-773 for TCF; and positions 333-351 for TXNIP. Commercially available rabbit polyclonal antibodies were used for BC-1514 (C15; Santa Cruz Biotechnology, Dallas, TX, USA), BC-1514 (W12; Santa Crus Biotechnology), CAMK2N1 (GenTex, Irvine, CA, USA), PJA2 (Abcam,
Cambridge, UK), and RPL5 (Abcam). A commercial mouse monoclonal antibody was used for CD1D (clone NOR3.2 [NOR3.2/13.17], Abcam).

\section{Tissue microarray}

The first tissue microarray was constructed with 231 consecutive surgical cases of invasive breast cancers from Kawasaki Medical School Hospital from September 2009 to December 2010. The first microarray contained 13 cases of invasive carcinoma with a micropapillary feature (ICMF: pure or mixed IMPC and ICNST with a focal micropapillary feature). The second tissue microarray was constructed with 63 cases of ICMF from January 2011 to December 2014. A KIN-2 system (Azumaya, Tokyo, Japan) with a 2-mm needle was used for the tissue microarray. For cases of ICMF, only the micropapillary area was sampled. ER and PgR were judged using 1\% cutoff [14]. For HER2, HercepTest 3+ or HercepTest $2+$ and FISH positive were regarded as positive [15].

\section{Immunohistochemistry}

Immunostaining was performed using an EnVision Plus kit (Dako, Glostrup, Denmark). We cut $4 \mu \mathrm{m}$ sections from the microarray tissue. After dewaxing and hydration, they were placed in a bath of hot citrate buffer, $\mathrm{pH}$ 6.0 at $95^{\circ} \mathrm{C}$ for $40 \mathrm{~min}$ for $\mathrm{BC}-1514$ (C15), BC-1514 (W12), CAMK2N1, CD1D, PJA2, SAMD13, TCF4 and

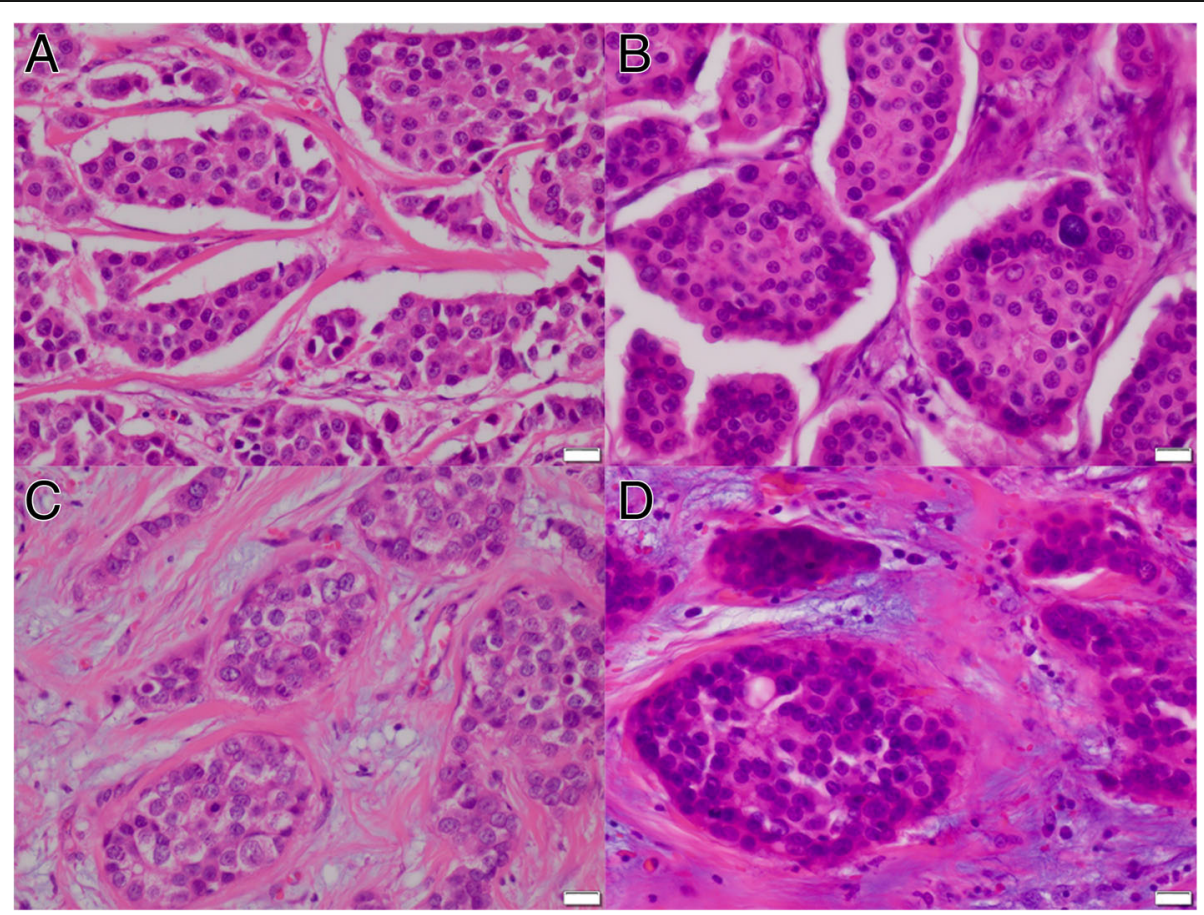

Fig. 1 Invasive micropapillary component of mixed IMPC, case 1 (a). Invasive micropapillary component of mixed IMPC, case 2 (b). Usual invasive carcinoma component of mixed IMPC, case 1 (c). Usual invasive carcinoma component of mixed IMPC, case 2 (d). Scale bars represent $20 \mu \mathrm{m}$ 
TXNIP, or of Target Retrieval Solution, pH 9.0 (Dako) for RPL5 at $95{ }^{\circ} \mathrm{C}$ for $40 \mathrm{~min}$. The sections were incubated with the primary antibodies for overnight at $4{ }^{\circ} \mathrm{C}$. The dilutions of primary antibodies were: 1:200 for BC-1514 (C15), 1:100 for BC-1514 (W12), 1:500 for CAMK2N1, 1:300 for CD1D, 1:100 for PJA2, and 1:1000 for RPL5. For SAMD13, TCF4, and TXNIP, the antibodies were used at $2 \mu \mathrm{g} / \mathrm{ml}$. The chromogen used was $3,3^{\prime}$-diaminobenzidine tetrachloride, and the sections were counterstained with hematoxylin.

Immunohistochemistry was analysed using a histoscore that was calculated by multiplying the positive area (\%) and intensity (0-3: 0 for negative, 1 for weak, 2 for moderate, and 3 for strong staining). Immunohistochemical analyses were evaluated in blinded manner.

\section{Statistical analyses}

Statistical analyses were performed using IBM SPSS Statistics for Windows (version 25; IBM Corp., Armonk, NY). $p<0.05$ was considered significant.

\section{Results}

\section{DNA microarray}

The commonly up- and downregulated factors in IMPC compared with ICNST by DNA microarray are shown in Table 1 and Table 2. BC-1514 (C21orf118) is the only gene that showed over threefold increasing expression in the IMPC area compared with the ICNST area. SAMD13, CAMK2N1, TCF4, TXNIP, RPL5, PJA2, and CD1d showed over threefold decreasing expression in the IMPC area compared with the ICNST area.

\section{Immunohistochemistry}

The immunohistochemical expression of BC-1514 (C15), BC-1514 (W12), CAMK2N1, CD1d, PJA2, RPL5, SAMD13, TCF4, and TXNIP are summarized in Table 3. BC-1514 (C15), BC-1514 (W12), CD1d, and PJA2 showed results concordant with the DNA microarray (Fig. 2A-D). CAMK2N1, RPL5, SAMD13, and TCF4 showed contradictory results, and TXNIP did not show a significant difference. We have not investigated these five genes further.

The expression of CD1d by ICNMF is similar to that of normal breast tissue $(p=0.373)$. However, expression of CD1d by ICMF is lower than that of normal breast tissue $(p=0.008)$. The expression of PJA2 by ICNMF is higher than that of normal breast tissue $(p<0.001)$. The expression of PJA2 by ICMF is similar to that of normal breast tissue $(p=0.259)$.

The clinicopathological analyses of BC-1514 (C15), BC-1514 (W12), CD1d, and PJA2 are shown in Table 4. Pathological $\mathrm{T}$ factor showed a significant positive correlation with BC-1514 (W12) $(p=0.015)$. Histological tumour grade and HER2 showed positive correlations with $\mathrm{BC}-1514$ (W12), respectively ( $p=0.009$ and $p=0.043$ ). The cases with a high Ki-67 index showed higher BC-1514 (C15) $(p=0.010), \mathrm{BC}-1514$ (W12) $(p=0.011)$, and CD1d $(p=0.036)$ than the cases with a low Ki-67 index.

\section{Survival analyses}

The median follow-up periods for disease-free survival was 87.8 months (range, 0.4 to 113.7 months) and for overall survival was 88.2 months (range, 0.4 to 119.27 months). The univariate Cox hazard survival analyses are shown in Table 5. pT, pN, and pathological stage were significant for

Table 1 DNA microarray data of mixed IMPC. Factors more commonly upregulated in the IMPC than ICNST areas. The ratio of the expression in IMPC area to the expression in ICNST area was shown as heat map. Red column represents over 3.0, pink represents 2.5 to 3.0 , yellow represents 2.1 to 2.4

\begin{tabular}{|llcc|}
\hline Symbol & Description & Case 1 & Case 2 \\
\hline C21orf118 & B lymphocyte activation-related protein BC-1514 & 20 & 4.1 \\
\hline SNAPIN & SNARE-associated protein Snapin & 9.4 & 2.4 \\
\hline LOC100294050 & - & 2.6 & 2.7 \\
\hline VPS37D & Vacuolar protein sorting-associated protein 37D & 2.5 & 2.1 \\
\hline KIF3C & Kinesin-like protein KIF3C & 2.4 & 2.9 \\
\hline C11orf9 & Uncharacterized protein C11orf9 & 2.4 & 2.7 \\
\hline C17orf62 & Uncharacterized protein C17orf62 & 2.3 & 2.3 \\
\hline DHsGV10001664 & & 2.3 & 2.3 \\
\hline SPSB4 & SPRY domain-containing SOCS box protein 4 (SSB-4) & 2.3 & 2.8 \\
\hline RAB11FIP3 & Rab11 family-interacting protein 3 & 2.2 & 2.1 \\
\hline CCR7 & C-C chemokine receptor type 7 Precursor & 2.2 & 2.1 \\
\hline KCNK4 & Potassium channel subfamily K member 4 & 2.1 & 2.7 \\
\hline ANP32C & $\begin{array}{l}\text { Acidic leucine-rich nuclear phosphoprotein 32 family } \\
\text { member C }\end{array}$ & 2.1 & 2.3 \\
\hline PCSK2 & Neuroendocrine convertase 2 Precursor & 2.1 & 3.3 \\
\hline
\end{tabular}


Table 2 DNA microarray data of mixed IMPC. Factors more commonly downregulated in the IMPC than ICNST areas. The ratio of the expression in IMPC area to the expression in ICNST area was shown as heat map. Violet column represents under 0.34, blue represents 0.34 to 0.4 , light blue represents 0.41 to 0.5

\begin{tabular}{|c|c|c|c|}
\hline Symbol & Description & Case 1 & Case 2 \\
\hline SAMD13 & Sterile alpha motif domain-containing protein 13 & 0.24 & 0.26 \\
\hline CAMK2N1 & $\begin{array}{l}\text { Calcium/calmodulin-dependent protein kinase II } \\
\text { inhibitor } 1\end{array}$ & 0.25 & 0.33 \\
\hline TCF4 & Transcription factor 4 & 0.26 & 0.2 \\
\hline TXNIP & Thioredoxin-interacting protein & 0.29 & 0.09 \\
\hline RPL5 & 60 S ribosomal protein L5 & 0.32 & 0.19 \\
\hline PJA2 & E3 ubiquitin-protein ligase Praja2 & 0.33 & 0.28 \\
\hline CD1D & T-cell surface glycoprotein CD1d Precursor & 0.33 & 0.13 \\
\hline GZMA & Granzyme A Precursor & 0.29 & 0.48 \\
\hline TTC7B & Tetratricopeptide repeat protein $7 \mathrm{~B}$ & 0.18 & 0.4 \\
\hline AC005726.6 & $\begin{array}{l}\text { Uncharacterized serine/threonine-protein kinase } \\
\text { SgK494 }\end{array}$ & 0.3 & 0.46 \\
\hline MYO9A & Myosin-IXa (Unconventional myosin-9a) & 0.33 & 0.44 \\
\hline NHS & Nance-Horan syndrome protein & 0.34 & 0.5 \\
\hline H2AFY & Core histone macro-H2A.1 & 0.35 & 0.39 \\
\hline PTN & Pleiotrophin Precursor (PTN) & 0.35 & 0.43 \\
\hline FNBP1 & Formin-binding protein 1 & 0.35 & 0.26 \\
\hline PIF1 & ATP-dependent DNA helicase PIF1 & 0.35 & 0.42 \\
\hline TCEA1 & Transcription elongation factor A protein 1 & 0.37 & 0.12 \\
\hline CBX6 & Chromobox protein homolog 6 & 0.37 & 0.44 \\
\hline PARP15 & Poly [ADP-ribose] polymerase 15 (PARP-15) & 0.37 & 0.42 \\
\hline PPP1R1B & Protein phosphatase 1 regulatory subunit $1 \mathrm{~B}$ & 0.39 & 0.47 \\
\hline PIK3R3 & $\begin{array}{l}\text { Phosphatidylinositol 3-kinase regulatory subunit } \\
\text { gamma }\end{array}$ & 0.39 & 0.47 \\
\hline C20orf6 & ESF1 homolog (ABT1-associated protein) & 0.41 & 0.2 \\
\hline ZNF425 & Zinc finger protein 425 & 0.42 & 0.47 \\
\hline FAM98A & Protein FAM98A & 0.43 & 0.31 \\
\hline C9orf93 & Uncharacterized protein C9orf93 & 0.43 & 0.49 \\
\hline RSRC2 & Arginine/serine-rich coiled-coil protein 2 & 0.43 & 0.39 \\
\hline CNN2 & Calponin-2 & 0.45 & 0.4 \\
\hline ZFP36L1 & Butyrate response factor 1 & 0.45 & 0.19 \\
\hline NUCKS1 & $\begin{array}{l}\text { Nuclear ubiquitous casein and cyclin-dependent } \\
\text { kinases substrate (P1) }\end{array}$ & 0.46 & 0.21 \\
\hline ZNF2 & Zinc finger protein 2 (Zinc finger protein 2.2 ) & 0.47 & 0.47 \\
\hline AGPS & Alkyldihydroxyacetonephosphate synthase & 0.47 & 0.46 \\
\hline CAMSAP1 & Calmodulin-regulated spectrin-associated protein 1 & 0.48 & 0.5 \\
\hline
\end{tabular}

disease free survival $(p=0.001,<0.001$ and 0.011$) . \mathrm{pT}, \mathrm{pN}$, stage, histological grade, and PgR were significant for overall survival $(p<0.001,0.004,<0.001,0.001$, and 0.005, respectively). BC-1514 (C15, W12), CD1d, and PJA2 did not show any significant difference for disease-free survival or overall survival. All patients with cases having high PJA2 survived without cancer recurrence (19 of 19) using the third quadrant as a cut-off value, but there was no significant difference ( $p=0.153$ for disease-free survival [Fig. 3] and $p=0.272$ for overall survival).

\section{Discussion}

CD1d-presents lipid antigens to activate natural killer $\mathrm{T}$ (NKT) cells, through the interaction with the T-cell receptor present on NKT membranes [16]. NKT cells display antitumour immune responses when activated by the synthetic glycosphingolipid, $\alpha$-galactosylceramide ( $\alpha$ GalCer) [16]. CD1d expression was reported in the intestines, liver, pancreas, kidney, uterus, skin, conjunctiva, thymus, tonsil, and breast [17]. We demonstrated that CD1d expression is lower in ICMF than in ICNMF 
Table 3 Immunohistochemical comparison of ICMF and ICNMF. Median (mean, IQR), ${ }^{*} p<0.05$

\begin{tabular}{llllll}
\hline & BC-1514 (C15) & BC-1514 (W12) & CAMK2N1 & CD1d & PJA2 \\
\hline ICMF & $200(218.5,200-300)$ & $200(243.6,200-300)$ & $200(192.1,200-200)$ & $80(90.6,35-170)$ & $100(134.5,90-200)$ \\
ICNMF & $180(139.1,90-200)$ & $200(210.9,180-300)$ & $90(83.0,80-100)$ & $95(114.1,80-190)$ & $200(164.3,100-200)$ \\
$p$ & $<0.001^{*}$ & $0.001^{*}$ & $<0.001^{*}$ & $0.006^{*}$ & $0.002^{*}$ \\
& RPL5 & SAMD13 & TCF4 & TXNIP & \\
ICMF & $300(233.4,300-300)$ & $90(95.8,60-100)$ & $200(241.5,200-300)$ & $100(126.9,80-200)$ \\
ICNMF & $40(58.9,10-90)$ & $80(67.3,10-90)$ & $200(219.2,200-300)$ & $90(114.4,80-190)$ \\
$p$ & $<0.001^{*}$ & $<0.001^{*}$ & $0.014^{*}$ & 0.109 & \\
\hline
\end{tabular}

and normal breast tissue. Downregulation of CD1d might be important for IMPC to avoid an NKT cell antitumour response. Hix et al. showed that downregulation of CD1d inhibited NKT-mediated antitumour immunity and promoted metastasis of breast cancer in vitro and in vivo [18]. Ascierto et al. reported that CD1d and CD96 were good prognostic factors for breast cancer [19], although we could not show a significant correlation between prognosis and CD1d expression. To improve the immune microenvironment, strategies such as $\alpha \mathrm{GalCer}$ [20] administration may be a therapeutic option for breast cancer.

PJA2, also known as PRAJA2, regulates the protein kinase A signal strength and duration in response to cAMP [21]. PJA2 increases the accumulation of ubiquitylated malignant fibrous histiocytoma amplified sequence 1, which promotes M1 macrophage polarization and M2 to M1 macrophage transformation [22]. M1 macrophage promotes antitumour immunity, while M2 macrophage promotes tumour progression [23]. We showed lower expression of PJA2 in ICMF than ICNMF by both DNA microarray and immunohistochemistry. In IMPC, M2 macrophage polarization by a shortage of PJA2 might facilitate tumour progression.

CD1d, PJA2, and granzyme A precursor also showed lower expression in IMPC than in ICNST in our DNA microarray screening. Granzyme A, an enzyme present in cytotoxic $\mathrm{T}$ lymphocytes, has tumouricidal activity $[24,25]$. The potential of IMPC to evade the immune system may be supported by multiple mechanisms.

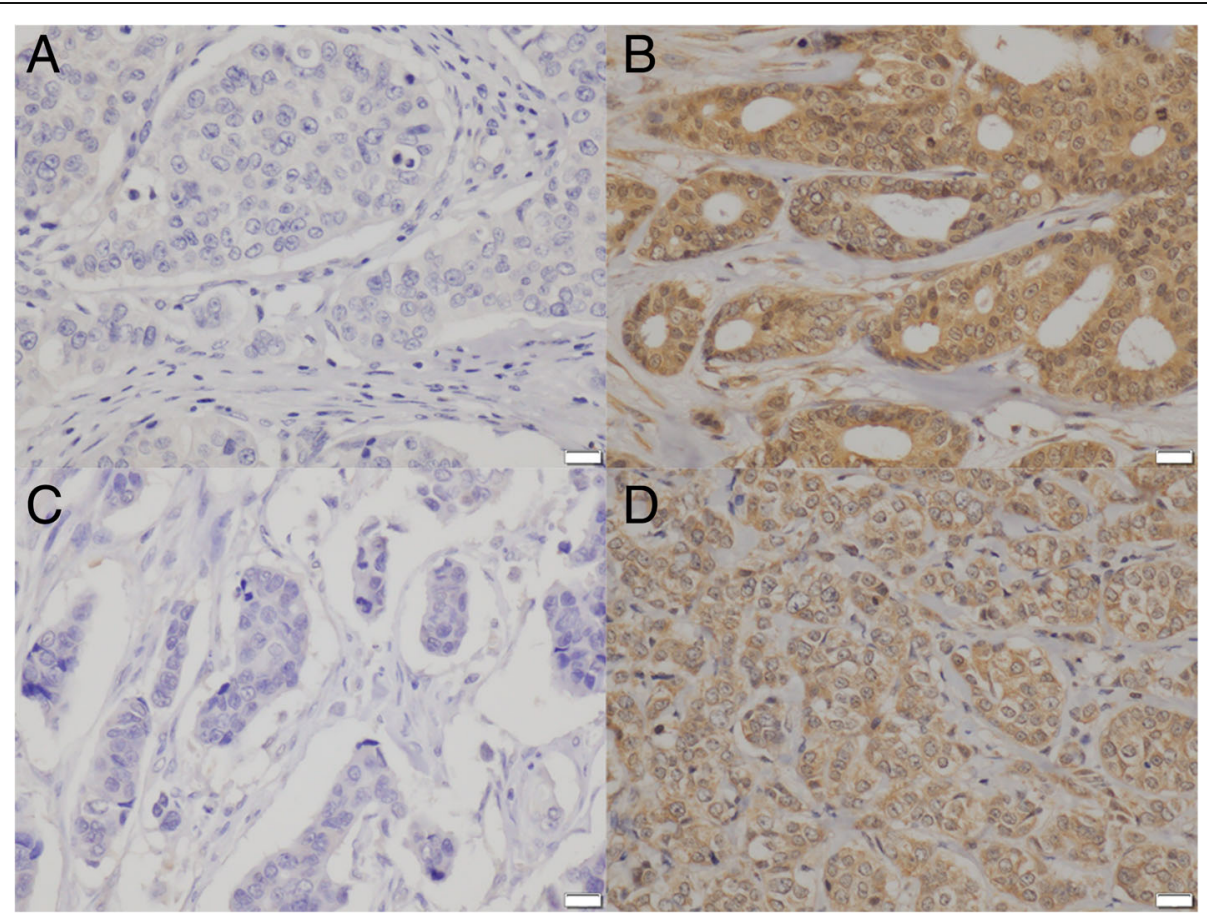

Fig. 2 Immunohistochemistry. Negative staining for CD1d in IMPC (a). Diffuse staining for CD1d in ICNST (b). Negative staining for PJA2 in IMPC (c). Diffuse staining for PJA2 in ICNST (d). Scale bars represent $20 \mu \mathrm{m}$ 
Table 4 The clinicopathological analyses of BC-1514 (C15), BC-1514 (W12), CD1d, and PJA2. Median (average, IQR), * $p<0.05$

\begin{tabular}{|c|c|c|c|c|c|c|c|c|c|c|}
\hline Parameter & & Cases (percentage) & BC-1514 (C15) & $P$ value & BC-1514 (W12) & $P$ value & CD1d & $P$ value & PJA2 & $P$ value \\
\hline \multicolumn{11}{|l|}{ Age } \\
\hline & $<55$ & $115(39.1)$ & $\begin{array}{l}180 \\
(160.7,90-200)\end{array}$ & 0.903 & $\begin{array}{l}200 \\
(212.4,200-300)\end{array}$ & 0.245 & $\begin{array}{l}90 \\
(98.2,60-180)\end{array}$ & 0.182 & $\begin{array}{l}180 \\
(157.3,90-200)\end{array}$ & 0.956 \\
\hline & $\geq 55$ & $179(60.9)$ & $\begin{array}{l}180 \\
(162.5,100-200)\end{array}$ & & $\begin{array}{l}200 \\
(225.7,200-300)\end{array}$ & & $\begin{array}{l}95 \\
(113.8,60-180)\end{array}$ & & $\begin{array}{l}180 \\
(155.5,90-200)\end{array}$ & \\
\hline \multicolumn{11}{|l|}{ pT } \\
\hline & $|-| \mid$ & $281(95.6)$ & $\begin{array}{l}180 \\
(161.1,90-200)\end{array}$ & 0.368 & $\begin{array}{l}200 \\
(218.2,200-300)\end{array}$ & $0.015^{*}$ & $\begin{array}{l}90 \\
(108.1,60-180)\end{array}$ & 0.413 & $\begin{array}{l}180 \\
(155.9,90-200)\end{array}$ & 0.445 \\
\hline & III-IV & $13(4.4)$ & $\begin{array}{l}200 \\
(177.5,100-300)\end{array}$ & & $\begin{array}{l}300 \\
(266.7,200-300)\end{array}$ & & $\begin{array}{l}90 \\
(100.0,10-180)\end{array}$ & & $\begin{array}{l}200 \\
(163.8,100-200)\end{array}$ & \\
\hline \multicolumn{11}{|l|}{$\mathrm{pN}$} \\
\hline & pNO & $192(65.3)$ & $\begin{array}{l}180 \\
(163.9,90-200)\end{array}$ & 0.709 & $\begin{array}{l}200 \\
(221.6,200-300)\end{array}$ & 0.979 & $\begin{array}{l}90 \\
(109.8,70-190)\end{array}$ & 0.466 & $\begin{array}{l}180 \\
(157.3,90-200)\end{array}$ & 0.99 \\
\hline & $\begin{array}{l}\text { pN1 or } \\
\text { above }\end{array}$ & $102(34.7)$ & $\begin{array}{l}180 \\
(157.9,95-200)\end{array}$ & & $\begin{array}{l}200 \\
(218.9,200-300)\end{array}$ & & $\begin{array}{l}90 \\
(103.9,40-180)\end{array}$ & & $\begin{array}{l}180 \\
(154.1,90-200)\end{array}$ & \\
\hline \multicolumn{11}{|c|}{ Distant metastasis } \\
\hline & Mo & $289(98.3)$ & $\begin{array}{l}180 \\
(160.5,90-200)\end{array}$ & 0.121 & $\begin{array}{l}200 \\
(219.4,200-300)\end{array}$ & 0.09 & $\begin{array}{l}90 \\
(108.1,60-180)\end{array}$ & 0.696 & $\begin{array}{l}180 \\
(155.8,90-200)\end{array}$ & 0.222 \\
\hline & M1 & $5(1.7)$ & $\begin{array}{l}250 \\
(215.0,105-300)\end{array}$ & & $\begin{array}{l}300 \\
(266.7,200-300)\end{array}$ & & $\begin{array}{l}90 \\
(92.0,45-135)\end{array}$ & & $\begin{array}{l}200 \\
(180.0,150-200)\end{array}$ & \\
\hline \multicolumn{11}{|l|}{ pStage } \\
\hline & $|-| \mid$ & $247(84.0)$ & $\begin{array}{l}180 \\
(161.5,90-200)\end{array}$ & 0.525 & $\begin{array}{l}200 \\
(220.1,200-300)\end{array}$ & 0.448 & $\begin{array}{l}90 \\
(107.2,60-180)\end{array}$ & 0.868 & $\begin{array}{l}180 \\
(155.3,90-200)\end{array}$ & 0.416 \\
\hline & III-IV & $47(16.0)$ & $\begin{array}{l}200 \\
(162.9,100-200)\end{array}$ & & $\begin{array}{l}200 \\
(222.5,200-300)\end{array}$ & & $\begin{array}{l}90 \\
(110.7,50-190)\end{array}$ & & $\begin{array}{l}200 \\
(161.0,100-200)\end{array}$ & \\
\hline \multicolumn{11}{|c|}{ Histological grade } \\
\hline & 1 or 2 & $230(78.2)$ & $\begin{array}{l}180 \\
(158.7,90-200)\end{array}$ & 0.176 & $\begin{array}{l}200 \\
(214.1,200-300)\end{array}$ & $0.009^{*}$ & $\begin{array}{l}90 \\
(107.4,60-180)\end{array}$ & 0.832 & $\begin{array}{l}180 \\
(158.2,95-200)\end{array}$ & 0.498 \\
\hline & 3 & $64(21.8)$ & $\begin{array}{l}200 \\
(172.6,100-200)\end{array}$ & & $\begin{array}{l}200 \\
(242.4,200-300)\end{array}$ & & $\begin{array}{l}95 \\
(109.2,60-180)\end{array}$ & & $\begin{array}{l}180 \\
(149.1,90-200)\end{array}$ & \\
\hline \multicolumn{11}{|c|}{ Lymphatic vessel invasion } \\
\hline & absent & $128(43.5)$ & $\begin{array}{l}180 \\
(156.1,90-200)\end{array}$ & 0.105 & $\begin{array}{l}200 \\
(218.8,200-300)\end{array}$ & 0.782 & $\begin{array}{l}90 \\
(110.8,70-190)\end{array}$ & 0.524 & $\begin{array}{l}180 \\
(156.4,90-200)\end{array}$ & 0.69 \\
\hline & present & $166(56.5)$ & $\begin{array}{l}200 \\
(166.3,100-200)\end{array}$ & & $\begin{array}{l}200 \\
(221.8,200-300)\end{array}$ & & $\begin{array}{l}90 \\
(105.4,50-180)\end{array}$ & & $\begin{array}{l}180 \\
(156.1,100-200)\end{array}$ & \\
\hline \multicolumn{11}{|c|}{ Blood vessel invasion } \\
\hline & absent & $243(82.7)$ & $\begin{array}{l}180 \\
(163.4,100-200)\end{array}$ & 0.57 & $\begin{array}{l}200 \\
(220.4,200-300)\end{array}$ & 0.895 & $\begin{array}{l}90 \\
(108.4,60-180)\end{array}$ & 0.836 & $\begin{array}{l}180 \\
(157.5,90-200)\end{array}$ & 0.543 \\
\hline & present & $51(17.3)$ & $\begin{array}{l}180 \\
(154.3,90-200)\end{array}$ & & $\begin{array}{l}200 \\
(220.9,200-300)\end{array}$ & & $\begin{array}{l}90 \\
(104.7,55-145)\end{array}$ & & $\begin{array}{l}180 \\
(150.0,100-200)\end{array}$ & \\
\hline \multicolumn{11}{|l|}{ ER } \\
\hline & negative & 48 (16.3) & $\begin{array}{l}200 \\
(157.4,90-200)\end{array}$ & 0.97 & $\begin{array}{l}200 \\
(241.7,200-300)\end{array}$ & 0.066 & $\begin{array}{l}95 \\
(121.7,90-180)\end{array}$ & 0.127 & $\begin{array}{l}200 \\
(165.9,95-200)\end{array}$ & 0.226 \\
\hline & positive & $246(83.7)$ & $\begin{array}{l}180 \\
(162.5,100-200)\end{array}$ & & $\begin{array}{l}200 \\
(217.1,200-300)\end{array}$ & & $\begin{array}{l}90 \\
(104.9,60-180)\end{array}$ & & $\begin{array}{l}180 \\
(154.3,90-200)\end{array}$ & \\
\hline \multicolumn{11}{|l|}{$\mathrm{PgR}$} \\
\hline & negative & 96 (32.7) & $\begin{array}{l}180 \\
(167.2,90-200)\end{array}$ & 0.605 & $\begin{array}{l}200 \\
(229.5,200-300)\end{array}$ & 0.208 & $\begin{array}{l}90 \\
(110.9,60-180)\end{array}$ & 0.632 & $\begin{array}{l}200 \\
(164.4,97.5-200)\end{array}$ & 0.105 \\
\hline & positive & $198(67.3)$ & $\begin{array}{l}180 \\
(159.5,100-200)\end{array}$ & & $\begin{array}{l}200 \\
(216.7,200-300)\end{array}$ & & $\begin{array}{l}90 \\
(106.3,60-190)\end{array}$ & & $\begin{array}{l}180 \\
(152.4,90-200)\end{array}$ & \\
\hline
\end{tabular}


Table 4 The clinicopathological analyses of BC-1514 (C15), BC-1514 (W12), CD1d, and PJA2. Median (average, IQR), * $p<0.05$ (Continued)

\begin{tabular}{|c|c|c|c|c|c|c|c|c|c|c|}
\hline Parameter & & Cases (percentage) & BC-1514 (C15) & $P$ value & BC-1514 (W12) & $P$ value & CD1d & $P$ value & PJA2 & $P$ value \\
\hline \multicolumn{11}{|l|}{ HER2 } \\
\hline & negative & $247(85.2)$ & $\begin{array}{l}180 \\
(160.1,90-200)\end{array}$ & 0.161 & $\begin{array}{l}200 \\
(216.8,200-300)\end{array}$ & $0.043^{*}$ & $\begin{array}{l}90 \\
(108.2,65-190)\end{array}$ & 0.471 & $\begin{array}{l}180 \\
(154.4,90-200)\end{array}$ & 0.207 \\
\hline & positive & $43(14.8)$ & $\begin{array}{l}200 \\
(176.0,100-200)\end{array}$ & & $\begin{array}{l}300 \\
(247.6,200-300)\end{array}$ & & $\begin{array}{l}92.5 \\
(108.3,40-180)\end{array}$ & & $\begin{array}{l}180 \\
(162.4,95-200)\end{array}$ & \\
\hline \multicolumn{11}{|l|}{$\mathrm{Ki}-67$} \\
\hline & $<14$ & $68(23.1)$ & $\begin{array}{l}160 \\
(136.8,90-200)\end{array}$ & $0.010^{*}$ & $\begin{array}{l}200 \\
(196.7,180-250)\end{array}$ & $0.011^{*}$ & $\begin{array}{l}90 \\
(91.7,55-92.5)\end{array}$ & $0.036^{*}$ & $\begin{array}{l}180 \\
(150.8,90-200)\end{array}$ & 0.603 \\
\hline & $\geq 14$ & $205(69.7)$ & $\begin{array}{l}180 \\
(158.9,95-200)\end{array}$ & & $\begin{array}{l}200 \\
(225.1,200-300)\end{array}$ & & $\begin{array}{l}95 \\
(112.8,60-190)\end{array}$ & & $\begin{array}{l}180 \\
(159.5,90-200)\end{array}$ & \\
\hline \multicolumn{11}{|c|}{ Triple negative } \\
\hline & no & $263(89.5)$ & $\begin{array}{l}180 \\
(164.4,100-200)\end{array}$ & 0.095 & $\begin{array}{l}200 \\
(219.4,200-300)\end{array}$ & 0.434 & $\begin{array}{l}90 \\
(106.3,60-180)\end{array}$ & 0.106 & $\begin{array}{l}180 \\
(154.9,90-200)\end{array}$ & 0.199 \\
\hline & yes & $31(10.5)$ & $\begin{array}{l}120 \\
(135.8,70-200)\end{array}$ & & $\begin{array}{l}200 \\
(231.2,200-300)\end{array}$ & & $\begin{array}{l}97.5 \\
(121.5,90-190)\end{array}$ & & $\begin{array}{l}200 \\
(167.8,90-200)\end{array}$ & \\
\hline
\end{tabular}

We demonstrated BC-1514 mRNA upregulation in IMPC and greater immunohistochemical expression in ICMF than ICNMF. However, the record of BC-1514 for Homo sapiens has been withdrawn by the National Center for Biotechnology Information because of insufficient evidence (https://www.ncbi.nlm.nih.gov/gene/378829, last accessed on June 6, 2018). Our immunohistochemistry for BC-1514 probably showed some cross reaction to an unknown substance.

One of the limitations of this study is that our DNA microarray data was obtained from only two mixed IMPC cases. We thought that the candidate markers for micropapillary feature were well narrowed down by just two cases. However, four (CAMK2N1, RPL5, SAMD13, and TCF4) of eight markers showed contradictory results, and TXNIP did not show a significant difference by immunohistochemistry. Additional DNA microarray would be promising to increase the accuracy, and should have improved the efficiency of our immunohistochemical study. Our DNA microarray data should be interpreted with caution.

Table 5 Univariate Cox proportional hazards model

\begin{tabular}{|c|c|c|c|c|c|}
\hline \multirow[b]{2}{*}{ Parameter } & \multirow[b]{2}{*}{ Criteria } & \multicolumn{2}{|c|}{ Disease free survival } & \multicolumn{2}{|c|}{ Overall survival } \\
\hline & & $p$ & HR $(95 \% \mathrm{Cl})$ & $p$ & HR $(95 \% \mathrm{Cl})$ \\
\hline Age & $<55$ vs. 55 or more & 0.556 & $0.803(0.386-1.669)$ & 0.322 & $0.594(0.212-1.667)$ \\
\hline pT & I-II vs. III-IV & $0.001^{*}$ & 6.135 (2.123-17.86) & $<0.001^{*}$ & $9.090(3.247-25.64)$ \\
\hline $\mathrm{pN}$ & 0 vs. $1-3$ & $<0.001^{*}$ & $4.950(2.252-10.87)$ & $0.004^{*}$ & $4.150(1.560-11.11)$ \\
\hline pStage & I-II vs. III-IV & $0.011^{*}$ & $2.793(1.267-6.135)$ & $<0.001^{*}$ & $5.587(2.212-14.08)$ \\
\hline Histological grade & $1 / 2$ vs. 3 & 0.360 & $1.464(0.648-3.307)$ & $0.001^{*}$ & $4.782(1.887-12.12)$ \\
\hline ER & Neg. vs. pos. & 0.934 & $0.960(0.366-2.519)$ & 0.528 & $0.698(0.230-2.123)$ \\
\hline $\mathrm{PgR}$ & Neg. vs. pos. & 0.142 & $0.770(0.578-1.202)$ & $0.005^{*}$ & $0.246(0.092-0.656)$ \\
\hline $\mathrm{Ki}-67$ & $<14 \%$ vs. $14 \%$ or more & 0.237 & $1.912(0.653-5.602)$ & 0.310 & $2.171(0.486-9.702)$ \\
\hline HER2 & Neg. vs. pos. & 0.600 & $1.377(0.416-4.553)$ & 0.779 & $1.194(0.335-4.132)$ \\
\hline Intrinsic subtype & Triple negative vs. others & 0.236 & $1.792(0.683-4.695)$ & 0.115 & $2.445(0.805-7.463)$ \\
\hline IMP & With IMP vs. without IMP & 0.064 & $2.032(0.958-4.309)$ & 0.308 & $1.669(0.624-4.462)$ \\
\hline BC-1514 (C15) & < 180 (median) vs. 180 or more & 0.476 & $1.342(0.597-3.016)$ & 0.304 & $1.823(0.580-5.726)$ \\
\hline BC-1514 (W12) & $<200$ (median) vs. 200 or more & 0.613 & $1.290(0.480-3.468)$ & 0.365 & $1.985(0.451-8.739)$ \\
\hline CD1d & $<90$ (median) vs. 90 or more & 0.674 & $0.843(0.381-1.866)$ & 0.391 & $0.609(0.196-1.890)$ \\
\hline PJA2 & < 180 (median) vs. 180 or more & 0.454 & $0.753(0.357-1.584)$ & 0.742 & $0.847(0.315-2.276)$ \\
\hline
\end{tabular}

${ }^{*} p<0.05$ 


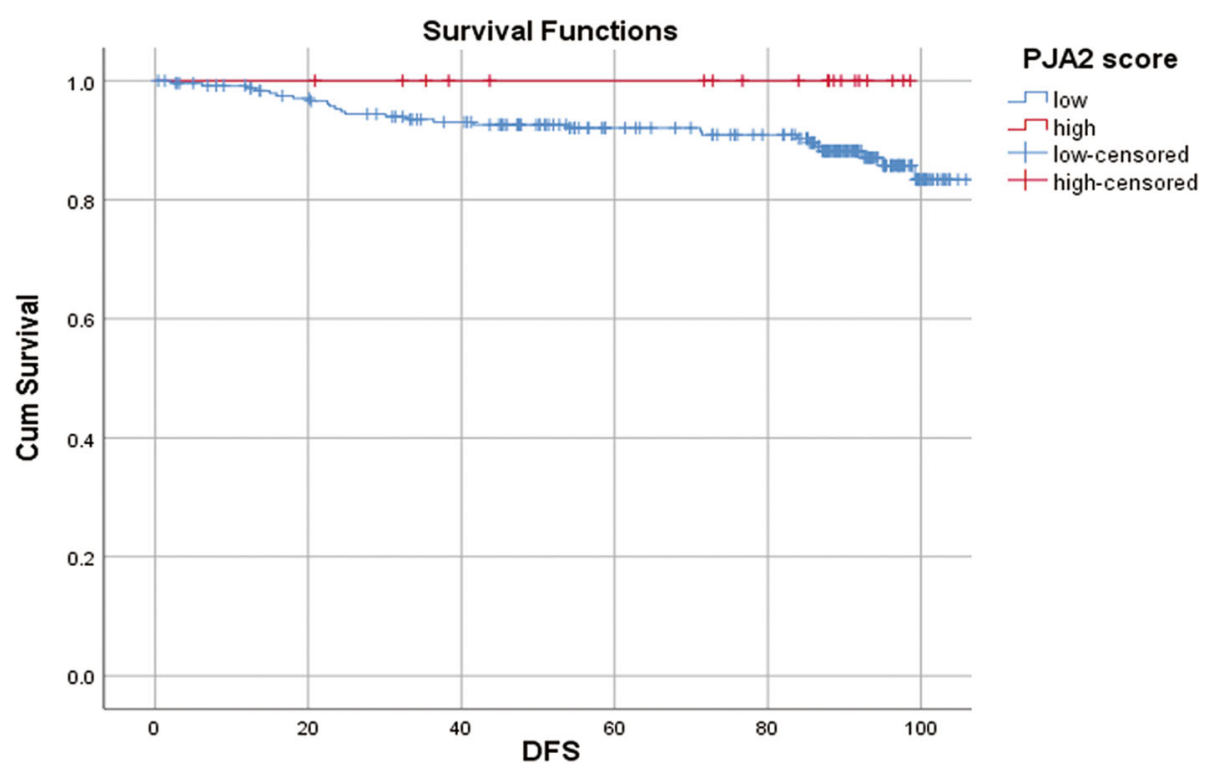

Fig. 3 Kaplan-Meier analysis of disease-free survival. No patients with cases having overexpression of PJA2 (> third quadrant) died or had tumour recurrence during the observed period $(p=0.153)$

\section{Conclusions}

Our present study suggests the CD1d- and PJA2-related tumour microenvironment might be crucial for IMPC. Further study of the immune microenvironment and micropapillary feature is warranted.

\section{Abbreviations}

ICMF: Invasive carcinoma with a micropapillary feature; ICNMF: Invasive carcinoma without a micropapillary feature; ICNST: Invasive carcinoma of no special type; IMPC: Invasive micropapillary carcinoma; IQR: Interquartile range

\section{Acknowledgments}

We thank Megumi Kuriyama for her technical assistance and Hiroko Murakami for her secretarial assistance.

\section{Funding}

This study was supported by grants from Kawasaki Medical School (no. 23K17) and JSPS KAKENHI (JP25460425) for data collection.

\section{Availability of data and materials}

All data generated or analysed during this study are included in this published article.

\section{Authors' contributions}

NK was responsible for DNA microarray, immunohistochemistry, and drafted the manuscript; JK and YK contributed clinical information. RY and TM contributed data analysis and interpretation. TM also supervised NK. All authors read and approved the manuscript.

\section{Ethics approval and consent to participate}

Written informed consent was obtained for use of retrospective tissue samples from the patients within this study. Ethnical approval was obtained from Institutional Review Board of Kawasaki Medical School ethics committee (approval number 909 and 2136).

\section{Consent for publication}

Not applicable.

\section{Competing interests}

The authors declare that they have no competing interests.

\section{Publisher's Note}

Springer Nature remains neutral with regard to jurisdictional claims in published maps and institutional affiliations.

\section{Author details}

'Department of Pathology, Kawasaki Medical School, Matsushima 577, Kurashiki, Okayama 701-0192, Japan. ${ }^{2}$ Department of Breast and Thyroid Surgery, Kawasaki Medical School, Kurashiki, Okayama, Japan. ${ }^{3}$ Department of Pathology and Laboratory Medicine, Kurume University Medical Center, Kurume, Fukuoka, Japan. ${ }^{4}$ Department of Pathology, Kurume University School of Medicine, Kurume, Fukuoka, Japan.

Received: 6 June 2018 Accepted: 13 December 2018

Published online: 16 January 2019

\section{References}

1. Siriaunkgul S, Tavassoli FA. Invasive micropapillary carcinoma of the breast. Mod Pathol. 1993;6(6):660-2.

2. Luna-More S, Gonzalez B, Acedo C, Rodrigo I, Luna C. Invasive micropapillary carcinoma of the breast. A new special type of invasive mammary carcinoma. Pathol Res Pract. 1994;190(7):668-74.

3. Li YS, Kaneko M, Sakamoto DG, Takeshima Y, Inai K. The reversed apical pattern of MUC1 expression is characteristics of invasive micropapillary carcinoma of the breast. Breast Cancer. 2006;13(1):58-63.

4. Wei J, Cui L, Liu F, Fan Y, Lang R, Gu F, Guo X, Tang P, Fu L. E-selectin and Sialyl Lewis $X$ expression is associated with lymph node metastasis of invasive micropapillary carcinoma of the breast. Int J Surg Pathol. 2010;18(3): 193-200.

5. Lepe M, Kalife ET, Ou J, Quddus MR, Singh K. Inside-out' p120 immunostaining pattern in invasive micropapillary carcinoma of the breast; additional unequivocal evidence of reversed polarity. Histopathology. 2017;70(5):832-4.

6. Guo X, Fan Y, Lang R, Gu F, Chen L, Cui L, Pringle GA, Zhang X. Fu L. tumor infiltrating lymphocytes differ in invasive micropapillary carcinoma and medullary carcinoma of breast. Mod Pathol. 2008;21(9):1101-7.

7. Yamaguchi R, Tanaka M, Kondo K, Yokoyama T, Kaneko Y, Yamaguchi M, Ogata Y, Nakashima O, Kage M, Yano H. Characteristic morphology of invasive micropapillary carcinoma of the breast: an immunohistochemical analysis. Jpn J Clin Oncol. 2010;40(8):781-7.

8. Liu F, Lang R, Wei J, Fan Y, Cui L, Gu F, Guo X, Pringle GA, Zhang X, Fu L. Increased expression of SDF-1/CXCR4 is associated with lymph node metastasis of invasive micropapillary carcinoma of the breast. Histopathology. 2009;54(6):741-50. 
9. Ren M, Liu F, Zhu Y, Li Y, Lang R, Fan Y, Gu F, Zhang X. Fu L. absence of caveolin-1 expression in carcinoma-associated fibroblasts of invasive micropapillary carcinoma of the breast predicts poor patient outcome. Virchows Arch. 2014:465(3):291-8.

10. Gong Y, Sun X, Huo L, Wiley EL, Rao MS. Expression of cell adhesion molecules, CD44s and E-cadherin, and microvessel density in invasive micropapillary carcinoma of the breast. Histopathology. 2005;46(1):24-30.

11. Meng F, Liu B, Xie G, Song Y, Zheng X, Qian X, Li S, Jia H, Zhang X, Zhang L, et al. Amplification and overexpression of PSCA at 8q24 in invasive micropapillary carcinoma of breast. Breast Cancer Res Treat. 2017;166(2): 383-92.

12. Wang XX, Liu BB, Wu X, Su D, Zhu Z. Fu L. loss of leucine zipper putative tumor suppressor 1 (LZTS1) expression contributes to lymph node metastasis of breast invasive micropapillary carcinoma. Pathol Oncol Res. 2015:21(4):1021-6.

13. Marchio C, Iravani M, Natrajan R, Lambros MB, Savage K, Tamber N, Fenwick K, Mackay A, Senetta R, Di Palma S, et al. Genomic and immunophenotypical characterization of pure micropapillary carcinomas of the breast. J Pathol. 2008;215(4):398-410.

14. Coates AS, Winer EP, Goldhirsch A, Gelber RD, Gnant M, Piccart-Gebhart M, Thurlimann B, Senn HJ, Panel M. Tailoring therapies--improving the management of early breast cancer: St Gallen international expert consensus on the primary therapy of early breast Cancer 2015. Ann Oncol. 2015;26(8):1533-46.

15. Muller KE, Marotti JD, Memoli VA, Wells WA, Tafe LJ. Impact of the 2013 ASCO/CAP HER2 guideline updates at an Academic Medical Center that performs primary HER2 FISH testing: increase in equivocal results and utility of reflex immunohistochemistry. Am J Clin Pathol. 2015;144(2):247-52.

16. Kawano T, Cui J, Koezuka Y, Toura I, Kaneko Y, Motoki K, Ueno H, Nakagawa $\mathrm{R}$, Sato $\mathrm{H}$, Kondo E, et al. CD1d-restricted and TCR-mediated activation of valpha14 NKT cells by glycosylceramides. Science. 1997;278(5343):1626-9.

17. Canchis PW, Bhan AK, Landau SB, Yang L, Balk SP, Blumberg RS. Tissue distribution of the non-polymorphic major histocompatibility complex class I-like molecule. CD1d Immunology. 1993;80(4):561-5.

18. Hix LM, Shi YH, Brutkiewicz RR, Stein PL, Wang CR, Zhang M. CD1dexpressing breast cancer cells modulate NKT cell-mediated antitumor immunity in a murine model of breast cancer metastasis. PLoS One 2011, 6(6):e20702.

19. Ascierto ML, Idowu MO, Zhao Y, Khalak H, Payne KK, Wang XY, Dumur $\mathrm{Cl}$, Bedognetti D, Tomei S, Ascierto PA, et al. Molecular signatures mostly associated with NK cells are predictive of relapse free survival in breast cancer patients. J Transl Med. 2013;11:145.

20. Veldt BJ, van der Vliet HJ, von Blomberg BM, van Vlierberghe $H$, Gerken $G$, Nishi N, Hayashi K, Scheper RJ, de Knegt RJ, van den Eertwegh AJ, et al. Randomized placebo controlled phase I/I trial of alpha-galactosylceramide for the treatment of chronic hepatitis C. J Hepatol. 2007;47(3):356-65.

21. Lignitto L, Carlucci A, Sepe M, Stefan E, Cuomo O, Nistico R, Scorziello A, Savoia C, Garbi C, Annunziato L, et al. Control of PKA stability and signalling by the RING ligase praja2. Nat Cell Biol. 2011;13(4):412-22.

22. Zhong J, Wang H, Chen W, Sun Z, Chen J, Xu Y, Weng M, Shi Q, Ma D, Miao C. Ubiquitylation of MFHAS1 by the ubiquitin ligase praja2 promotes M1 macrophage polarization by activating JNK and p38 pathways. Cell Death Dis. 2017;8(5):e2763.

23. Sica A, Mantovani A. Macrophage plasticity and polarization: in vivo veritas J Clin Invest. 2012;122(3):787-95.

24. Masson D, Zamai M, Tschopp J. Identification of granzyme a isolated from cytotoxic T-lymphocyte-granules as one of the proteases encoded by $\mathrm{CTL}$ specific genes. FEBS Lett. 1986;208(1):84-8.

25. Legrand F, Driss V, Delbeke M, Loiseau S, Hermann E, Dombrowicz D, Capron M. Human eosinophils exert TNF-alpha and granzyme A-mediated tumoricidal activity toward colon carcinoma cells. J Immunol. 2010;185(12): 7443-51.

Ready to submit your research? Choose BMC and benefit from:

- fast, convenient online submission

- thorough peer review by experienced researchers in your field

- rapid publication on acceptance

- support for research data, including large and complex data types

- gold Open Access which fosters wider collaboration and increased citations

- maximum visibility for your research: over $100 \mathrm{M}$ website views per year

At BMC, research is always in progress.

Learn more biomedcentral.com/submissions 